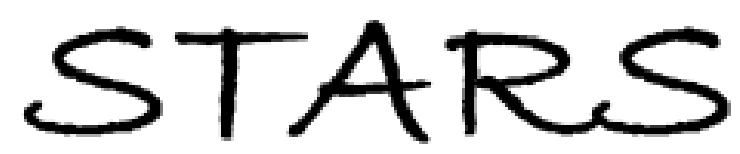

University of Central Florida

STARS

$1-1-2002$

\title{
Theoretically exact filtered backprojection-type inversion algorithm for spiral CT
}

\author{
Alexander Katsevich \\ University of Central Florida
}

Find similar works at: https://stars.library.ucf.edu/facultybib2000 University of Central Florida Libraries http://library.ucf.edu

This Article is brought to you for free and open access by the Faculty Bibliography at STARS. It has been accepted for inclusion in Faculty Bibliography 2000 s by an authorized administrator of STARS. For more information, please contactSTARS@ucf.edu.

\section{Recommended Citation}

Katsevich, Alexander, "Theoretically exact filtered backprojection-type inversion algorithm for spiral CT" (2002). Faculty Bibliography 2000s. 3280.

https://stars.library.ucf.edu/facultybib2000/3280

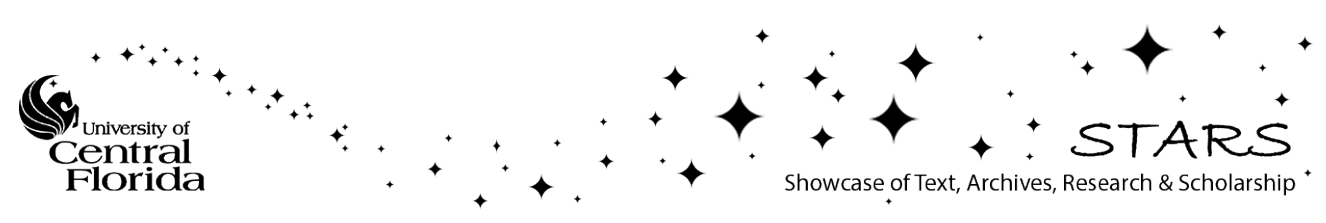




\title{
THEORETICALLY EXACT FILTERED BACKPROJECTION-TYPE INVERSION ALGORITHM FOR SPIRAL CT*
}

\author{
ALEXANDER KATSEVICH ${ }^{\dagger}$
}

\begin{abstract}
Proposed is a theoretically exact formula for inversion of data obtained by a spiral computed tomography (CT) scan with a two-dimensional detector array. The detector array is supposed to be of limited extent in the axial direction. The main property of the formula is that it can be implemented in a truly filtered backprojection fashion. First, one performs shift-invariant filtering of a derivative of the cone beam projections, and, second, the result is backprojected in order to form an image. Another property is that the formula solves the so-called long object problem. Limitations of the algorithm are discussed. Results of numerical experiments are presented.
\end{abstract}

Key words. cone beam, spiral tomography, theoretically exact reconstruction, filtered backprojection algorithm

AMS subject classifications. 44A12, 65R10, 92C55

PII. S0036139901387186

1. Introduction. Spiral computed tomography $(\mathrm{CT})$ involves continuous data acquisition throughout the volume of interest by simultaneously moving the patient through the gantry while the x-ray source rotates. Spiral CT has numerous advantages over conventional $\mathrm{CT}$ and is now a standard medical imaging modality. In the past decade it became clear that spiral CT can be significantly improved if one uses two-dimensional detector arrays instead of one-dimensional ones. This lead to the development of scanners with multiple detector rows. At the present time, scanners with four and eight detector rows are commercially available. It appears that as the technology advances further, scanners with even higher number of detector rows will emerge. On the other hand, accurate and efficient image reconstruction from the data provided by such scanners is very challenging because there does not exist a theoretically exact and efficient reconstruction formula. Several approaches for image reconstruction have been proposed. They can be classified into two groups: theoretically exact and approximate. See [TD00] for a recent review of available algorithms. Most exact algorithms are based on computing the Radon transform for a given plane by partitioning the plane in a manner determined by the spiral path of the x-ray source [Tam95, Tam97, KS97, $\left.\mathrm{SNS}^{+} 00\right]$. Even though exact algorithms are more accurate, they are computationally quite intensive and require keeping considerable amount of cone beam $(\mathrm{CB})$ projections in memory. Approximate algorithms are much more efficient (see, e.g., [KND98, NKD98, DNK00, Kat02] for several most recent techniques) but produce artifacts, which can be significant under unfavorable circumstances. Despite the significant progress achieved in recent years, it appears that no algorithm which would be both efficient and theoretically exact have been proposed in the literature so far.

In this paper we propose the first theoretically exact inversion formula which is truly of the filtered backprojection (FBP) type. This means that the formula can

\footnotetext{
${ }^{*}$ Received by the editors April 3, 2001; accepted for publication (in revised form) January 11, 2002; published electronically July 3, 2002. This research was supported in part by NSF grant DMS9704285.

http://www.siam.org/journals/siap/62-6/38718.html

†Department of Mathematics, University of Central Florida, Orlando, FL 32816-1364 (akatsevi@ pegasus.cc.ucf.edu).
} 

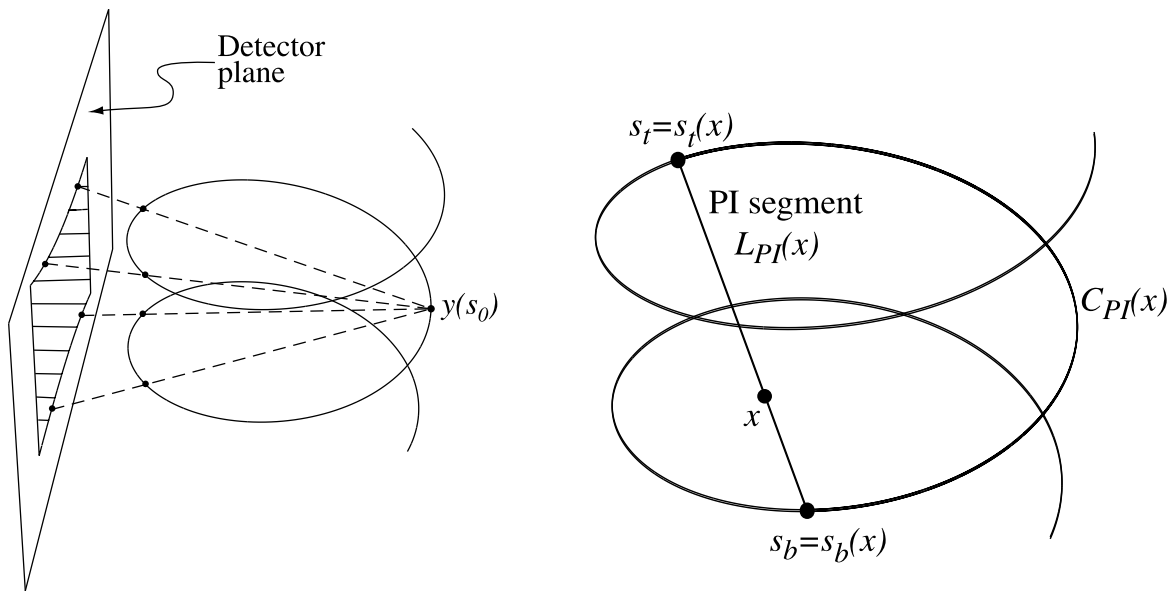

FIG. 1. Illustration of the smallest detector array required for exact reconstruction (left panel) and illustration of a PI line (right panel).

be numerically implemented in two steps. First, one performs shift-invariant filtering of a derivative of the $\mathrm{CB}$ projections, and, second, the result is backprojected in order to form an image. The price to pay for this efficient structure is that the algorithm requires a detector array wider than the theoretically minimum one. Also, the algorithm is applicable if the radius of support of the patient inside the gantry is not too big (not greater than $\approx 0.62 \times$ radius of gantry). Clearly, this limitation is not a big problem in most cases, for example, when one scans the head or an extremity of a patient.

In section 2 we derive the inversion formula. In section 3 we show that the resulting algorithm is of the FBP type and present the results of two numerical experiments.

2. Inversion formula. First we introduce the necessary notations. Let

$$
C:=\left\{y \in \mathbb{R}^{3}: y_{1}=R \cos (s), y_{2}=R \sin (s), y_{3}=s(h / 2 \pi), s \in \mathbb{R}\right\},
$$

where $h>0$ is a spiral, and $U$ is an open set strictly inside the spiral:

$$
\bar{U} \subset\left\{x \in \mathbb{R}^{3}: x_{1}^{2}+x_{2}^{2}<r^{2}\right\}, \quad 0<r<R .
$$

$S^{2}$ is the unit sphere in $\mathbb{R}^{3}$, and

$$
\begin{aligned}
& D_{f}(y, \beta):=\int_{0}^{\infty} f(y+\beta t) d t, \beta \in S^{2} \\
& \beta(s, x):=\frac{x-y(s)}{|x-y(s)|}, \quad x \in U, s \in \mathbb{R}, \Pi(x, \xi):=\left\{y \in \mathbb{R}^{3}:(y-x) \cdot \xi=0\right\}
\end{aligned}
$$

that is, $D_{f}(y, \beta)$ is the CB transform of $f$. Given $(x, \xi) \in U \times\left(\mathbb{R}^{3} \backslash 0\right)$, let $s_{j}=$ $s_{j}(\xi, \xi \cdot x), j=1,2, \ldots$, denote finitely many points of intersection of the plane $\Pi(x, \xi)$ with $C$. Also, $\dot{y}(s):=d y / d s$. As was shown in $\left[\mathrm{D}^{+} 97\right.$, DNK00], any point strictly inside the spiral belongs to one and only one parametric interval (PI) segment. Recall that a PI segment is a segment of line endpoints which are located on the spiral and separated by less than one pitch in the axial direction (see Figure 1, right panel). Let $s=s_{b}(x)$ and $s=s_{t}(x)$ denote values of the parameter corresponding to the 
endpoints of the PI segment containing $x$. We will call $I_{P I}(x):=\left[s_{b}(x), s_{t}(x)\right]$ the PI parametric interval. The part of the spiral corresponding to $I_{P I}(x)$ will be denoted $C_{P I}(x)$. Also, inside the PI there exists $\check{s}=\check{s}(x)$ such that the plane through $y(\check{s})$ and parallel to $\dot{y}(\check{s}), \ddot{y}(\check{s})$, contains $x$.

Fix $x \in U$. It is clear that any plane through $x$ intersects $C_{P I}(x)$ at least at one point. Introduce the following sets:

$$
\begin{aligned}
\operatorname{Crit}(x) & =\left\{\xi \in \mathbb{R}^{3} \backslash 0: \Pi(x, \xi) \text { contains } y\left(s_{b}(x)\right), y\left(s_{t}(x)\right)\right. \text { or } \\
& \left.\Pi(x, \xi) \text { is tangent to } C_{P I}(x)\right\} \cup\{0\}, \\
\Xi_{1}(x)= & \left\{\xi \in \mathbb{R}^{3}: \xi \notin \operatorname{Crit}(x) \text { and } \Pi(x, \xi) \cap C_{P I}(x) \text { contains one point }\right\}, \\
\Xi_{3}(x)= & \mathbb{R}^{3} \backslash\left\{\Xi_{1}(x) \cup \operatorname{Crit}(x)\right\} .
\end{aligned}
$$

By construction, the sets $\operatorname{Crit}(x), \Xi_{1,3}(x)$ are pairwise disjoint, their union is all of $\mathbb{R}^{3}, \operatorname{Crit}(x)$ is closed and has Lebesgue measure zero, and $\Xi_{1,3}(x)$ are open.

Denote

$$
e_{1}(s, x):=\frac{[\beta(s, x) \times \dot{y}(s)] \times \beta(s, x)}{|[\beta(s, x) \times \dot{y}(s)] \times \beta(s, x)|} .
$$

By construction, $e_{1}(s, x)$ is a unit vector in the plane through $y(s)$ and is spanned by $\beta(s, x), \dot{y}(s)$. Moreover, $e_{1}(s, x)$ is perpendicular to $\beta(s, x)$. For convenience, here and in the rest of the paper, we think of vectors $\beta(s, x), e_{1}(s, x), e_{2}(s, x)$ (to be defined below) and their linear combinations as if they are attached to $y(s)$.

Given $y(s), s \in\left(s_{b}(x), s_{t}(x)\right) \backslash\{\check{s}(x)\}$, find $s_{\text {tan }} \in I_{P I}(x), s_{\text {tan }} \neq s$, such that the plane through $x, y(s)$, and $y\left(s_{t a n}\right)$ is tangent to $C_{P I}(x)$ at $y\left(s_{t a n}\right)$. This is equivalent to solving

$$
\left[\left(x-y\left(s_{\text {tan }}\right)\right) \times(x-y(s))\right] \cdot \dot{y}\left(s_{\text {tan }}\right)=0, s_{\text {tan }} \neq s .
$$

Existence and uniqueness of the solution $s_{\tan } \in I_{P I}(x)$ to $(2.7)$ is shown below (see the second paragraph following (2.24)). Also, we will show below (see (2.35) and the argument near it) that $s_{\tan }(s, x)$ is $C^{\infty}$ with respect to $s$ on $\left(s_{b}(x), s_{t}(x)\right) \backslash\{\check{s}(x)\}$ and is made continuous on $\left[s_{b}(x), s_{t}(x)\right]$ by setting

$$
s_{\text {tan }}(s, x)= \begin{cases}s_{t}(x), & s=s_{b}(x), \\ \check{s}(x), & s=\check{s}(x), \\ s_{b}(x), & s=s_{t}(x) .\end{cases}
$$

Once $s_{\tan }=s_{\tan }(s, x)$ has been found, denote similarly to $(2.6)$

$$
\begin{aligned}
e_{2}(s, x) & :=\frac{[\beta(s, x) \times \Theta] \times \beta(s, x)}{|[\beta(s, x) \times \Theta] \times \beta(s, x)|}, \\
\Theta & = \begin{cases}\operatorname{sgn}\left(s-s_{\text {tan }}(s, x)\right) \beta\left(s_{\text {tan }}, x\right), & s \in\left(s_{b}(x), s_{t}(x)\right) \backslash\{\check{s}(x)\}, \\
\dot{y}\left(s_{\text {tan }}\right), & s \in\left\{s_{b}(x), \check{s}(x), s_{t}(x)\right\} .\end{cases}
\end{aligned}
$$

By construction, $e_{2}(s, x)$ is a unit vector in the plane through $x, y(s)$ and is a tangent to $C_{P I}(x)$ at $y\left(s_{\tan }\right)$. In addition, $e_{2}(s, x)$ is perpendicular to $\beta(s, x)$. Using $(2.8)$ and the inequalities $s_{\tan }(s, x)>\check{s}(x)$ if $s<\check{s}(x), s_{\tan }(s, x)<\check{s}(x)$ if $s>\check{s}(x)$ (see (2.39) below), we conclude that $e_{2}(s, x)$ is continuous with respect to $s$ on $\left[s_{b}(x), s_{t}(x)\right]$. 
For $f \in C_{0}^{\infty}(U)$ and $k=1,2$ define

$$
\begin{aligned}
\left(\mathcal{B}_{k} f\right)(x):=-\frac{1}{2 \pi^{2}} & \int_{I_{P I}(x)} \frac{1}{|x-y(s)|} \\
& \times\left.\int_{0}^{2 \pi} \frac{\partial}{\partial q} D_{f}\left(y(q), \cos \gamma \beta(s, x)+\sin \gamma e_{k}(s, x)\right)\right|_{q=s} \frac{d \gamma}{\sin \gamma} d s
\end{aligned}
$$

where $e_{1,2}(s, x)$ are given by (2.6) and (2.9). Our main result is the following theorem.

THEOREM 2.1. The operators $\mathcal{B}_{k}, k=1,2$, can be written in the form

$$
\left(\mathcal{B}_{k} f\right)(x)=\frac{1}{(2 \pi)^{3}} \int_{\mathbb{R}^{3}} B_{k}(x, \xi) \tilde{f}(\xi) e^{-i \xi \cdot x} d \xi,
$$

where for each $x \in U, B_{k}(x, \xi) \in L^{\infty}\left(\mathbb{R}^{3}\right)$ with respect to $\xi$ and

$$
B_{1}(x, \xi)=\left\{\begin{array}{ll}
1, & \xi \in \Xi_{1}(x), \\
3, & \xi \in \Xi_{3}(x),
\end{array} \quad B_{2}(x, \xi)= \begin{cases}1, & \xi \in \Xi_{1}(x), \\
-1, & \xi \in \Xi_{3}(x) .\end{cases}\right.
$$

Since the set $\operatorname{Crit}(x)$ has Lebesgue measure zero, (2.12) immediately implies the following inversion formula.

COROLlaRY 2.2. Under the assumptions of Theorem 2.1,

$$
f=\frac{1}{2}\left(\mathcal{B}_{1} f+\mathcal{B}_{2} f\right)
$$

An important feature of the double integral in (2.10) is that for each $x \in U$ the integral with respect to $s$ is confined only to the theoretically minimal portion of the parametric interval $I_{P I}(x)$. Moreover, it will be shown later (see section 3) that (2.11) requires only a finite detector array regardless of how long the support of $f$ is along the axial direction. This implies that inversion formula (2.13) solves the so-called long object problem (see [DNK00] for a definition).

Proof of Theorem 2.1. Let $x \in U$ be fixed. Consider the integral with respect to $\gamma$ in $(2.10)$ :

$$
\begin{aligned}
& \left.\int_{0}^{2 \pi} \frac{\partial}{\partial q} \int_{0}^{\infty} f\left(y(q)+t\left(\cos \gamma \beta(s, x)+\sin \gamma e_{k}(s, x)\right)\right)\right|_{q=s} \frac{1}{t \sin \gamma} t d t d \gamma \\
& =\left.\int_{\mathbb{R}^{2}} \frac{\partial}{\partial q} f(y(q)+u)\right|_{q=s} \frac{1}{u \cdot e_{k}(s, x)} d u \\
& =\left.\frac{1}{(2 \pi)^{3}} \int_{\mathbb{R}^{3}} \tilde{f}(\xi) \int_{\mathbb{R}^{2}} \frac{\partial}{\partial q} e^{-i \xi \cdot(y(q)+u)}\right|_{q=s} \frac{1}{u \cdot e_{k}(s, x)} d u d \xi \\
& =\frac{1}{(2 \pi)^{3}} \int_{\mathbb{R}^{3}} \tilde{f}(\xi)(-i \xi \cdot \dot{y}(s)) e^{-i \xi \cdot y(s)}\left[\int_{\mathbb{R}} e^{-i \xi_{1} u_{1}} d u_{1} \int_{\mathbb{R}} e^{-i \xi_{2} u_{2}} \frac{d u_{2}}{u_{2}}\right] d \xi \\
& =\frac{1}{(2 \pi)^{3}} \int_{\mathbb{R}^{3}} \tilde{f}(\xi)(-i \xi \cdot \dot{y}(s)) e^{-i \xi \cdot y(s)} 2 \pi \delta\left(\xi_{1}\right)\left(-i \pi \operatorname{sgn} \xi_{2}\right) d \xi \\
& =\frac{-|x-y(s)|}{4 \pi} \int_{\mathbb{R}^{3}} \tilde{f}(\xi)(\xi \cdot \dot{y}(s)) e^{-i \xi \cdot y(s)} \delta(\xi \cdot(x-y(s))) \operatorname{sgn}\left(\xi \cdot e_{k}(s, x)\right) d \xi
\end{aligned}
$$

where $d u$ is the Lebesgue measure on the plane through the origin and is parallel to $\beta(s, x), e_{k}(s, x)$. In (2.14) we have assumed without loss of generality that the $\xi_{1}$-axis is parallel to $\beta(s, x)$ and the $\xi_{2}$-axis is parallel to $e_{k}(s, x)$. 
Since (2.14) is the starting point of the entire proof, let us briefly discuss regularization of the divergent integrals occurring there. Write the integral with respect to $\gamma$ in (2.10) in the form

$$
\left.\int_{|\gamma|<2 \pi-a} \frac{\partial}{\partial q} \frac{D_{f}\left(y(q), \cos \gamma \beta(s, x)+\sin \gamma e_{k}(s, x)\right)-D_{f}(y(q), \beta(s, x))}{\sin \gamma}\right|_{q=s} d \gamma,
$$

where $a>0$ is small enough, so that the wedge $|\gamma|<2 \pi-a$ with vertex at $y(s)$ contains $\operatorname{supp} f$. Clearly, $\partial / \partial q$ can be moved outside the integral. For convenience assume for a moment that the $x_{1}$ and $x_{2}$ axes are parallel to $\beta(s, x)$ and $e_{k}(s, x)$, respectively. Similarly to $(2.14),(2.15)$ transforms to

$$
\begin{aligned}
& \left.\frac{\partial}{\partial q} \lim _{\epsilon \rightarrow 0^{+}} \iint_{\epsilon\left|u_{1}\right| \leq\left|u_{2}\right|} f\left(y_{1}(q)+u_{1}, y_{2}(q)+u_{2}, y_{3}(q)\right) \frac{d u_{2}}{u_{2}} d u_{1}\right|_{q=s} \\
& =\left.\frac{\partial}{\partial q} \lim _{\epsilon \rightarrow 0^{+}} \iint_{\left|u_{2}\right|>\epsilon} f\left(y_{1}(q)+u_{1}, y_{2}(q)+u_{2}, y_{3}(q)\right) \frac{d u_{2}}{u_{2}} d u_{1}\right|_{q=s},
\end{aligned}
$$

which is easily seen to be equivalent to the last integral in (2.14).

Pick any $\delta_{1} \in C_{0}^{\infty}(\mathbb{R}), \delta_{1}(t) \geq 0, \int \delta_{1}(t) d t=1$, and define $\delta_{\epsilon}(t)=\epsilon^{-1} \delta_{1}(t / \epsilon), \epsilon>$ 0 . Replacing $\delta$ and sgn by $\delta_{\epsilon}$ and $\operatorname{sgn}_{\epsilon}=\operatorname{sgn} * \delta_{\epsilon}$, respectively, in (2.14) we get

$$
A(s, x)=\lim _{\epsilon \rightarrow 0^{+}} \int_{\mathbb{R}^{3}} \tilde{f}(\xi)(\xi \cdot \dot{y}(s)) \delta_{\epsilon}(\xi \cdot(x-y(s))) \operatorname{sgn}_{\epsilon}\left(\xi \cdot e_{k}(s, x)\right) e^{-i \xi \cdot y(s)} d \xi,
$$

where $A(s, x)$ is the last integral in (2.14). Substituting into (2.10) we get

$$
\left(\mathcal{B}_{k} f\right)(x)=\frac{1}{(2 \pi)^{3}} \int_{I_{P I}(x)} \lim _{\epsilon \rightarrow 0^{+}} A_{\epsilon}(s, x) d s,
$$

where $A_{\epsilon}(s, x)$ is the integral on the right in (2.17). Since $\tilde{f} \in \mathcal{S}\left(\mathbb{R}^{3}\right)$ and $x-y(s) \perp$ $e_{k}(s, x)$, it is easy to see that $A_{\epsilon}(s, x)$ is uniformly bounded with respect to $s \in I_{P I}(x)$ as $\epsilon \rightarrow 0^{+}$. Hence, using the Lebesgue dominated convergence theorem and changing the order of integration,

$$
\begin{aligned}
\left(\mathcal{B}_{k} f\right)(x) & =\frac{1}{(2 \pi)^{3}} \lim _{\epsilon \rightarrow 0^{+}} \int_{\mathbb{R}^{3}} \tilde{f}(\xi) G_{\epsilon}(x, \xi) d \xi, \\
G_{\epsilon}(x, \xi) & :=\int_{I_{P I}(x)}(\xi \cdot \dot{y}(s)) \delta_{\epsilon}(\xi \cdot(x-y(s))) \operatorname{sgn}_{\epsilon}\left(\xi \cdot e_{k}(s, x)\right) e^{-i \xi \cdot y(s)} d s .
\end{aligned}
$$

Clearly, $G_{\epsilon}(x, \xi=0)=0$. We will show that $\left|G_{\epsilon}(x, \xi)\right|<c, \xi \neq 0$, for some $c>0$ and all $\epsilon>0$. Indeed, let $s=q_{k} \in I_{P I}(x), q_{1}<q_{2}<\ldots$, be the roots of the equation $\xi \cdot \dot{y}(s)=0$. Obviously the number of such roots is uniformly bounded with respect to $\xi \in \mathbb{R}^{3} \backslash 0$. Say there are no more than $K$ roots. Then

$$
\begin{aligned}
\left|G_{\epsilon}(x, \xi)\right| & \leq \int_{I_{P I}(x)} \delta_{\epsilon}(\xi \cdot(x-y(s)))|\xi \cdot \dot{y}(s)| d s \\
& \leq\left(\int_{s_{b}}^{q_{1}}+\sum_{k=1}^{K-1} \int_{q_{k}}^{q_{k+1}}+\int_{q_{K}}^{s_{t}}\right) \delta_{\epsilon}(\xi \cdot(x-y(s)))(\xi \cdot \dot{y}(s)) d s \operatorname{sgn}\left(\xi \cdot \dot{y}\left(q_{k}^{*}\right)\right),
\end{aligned}
$$


where $q_{k}^{*}$ is the midpoint of the corresponding interval of integration. Each term in the summation in (2.20) is bounded because

$$
\begin{aligned}
& \int_{q_{k}}^{q_{k+1}} \delta_{\epsilon}(\xi \cdot(x-y(s)))(\xi \cdot \dot{y}(s)) d s \operatorname{sgn}\left(\xi \cdot \dot{y}\left(q_{k}^{*}\right)\right) \\
& \leq \int_{t=\xi \cdot\left(x-y\left(q_{k}\right)\right)}^{t=\xi \cdot\left(x-y\left(q_{k+1}\right)\right)} \delta_{\epsilon}(\xi \cdot x-t) d t \operatorname{sgn}\left(\xi \cdot \dot{y}\left(q_{k}^{*}\right)\right) \leq \int \delta_{\epsilon}(\xi \cdot x-t) d t=1,
\end{aligned}
$$

and (2.20), (2.21) imply $\left|G_{\epsilon}(x, \xi)\right|<K+1$. Take any $\xi \notin C r i t(x)$. An easy calculation shows

$$
\begin{aligned}
\lim _{\epsilon \rightarrow 0^{+}} G_{\epsilon}(x, \xi) & =e^{-i \xi \cdot x} B_{k}(x, \xi), \\
B_{k}(x, \xi) & =\sum_{s_{j} \in I_{P I}(x)} \operatorname{sgn}\left(\xi \cdot \dot{y}\left(s_{j}\right)\right) \operatorname{sgn}\left(\xi \cdot e_{k}\left(s_{j}, x\right)\right), \quad k=1,2 .
\end{aligned}
$$

Recall that $s_{j}=s_{j}(\xi, \xi \cdot x), j=1,2, \ldots$, denote parameter values corresponding to the points of intersection of the plane $\Pi(x, \xi)$ with the spiral and are found by solving $\xi \cdot(x-y(s))=0$. Here we have used that $\xi \cdot\left(x-y\left(s_{j}\right)\right)=0$ implies $\xi \cdot \dot{y}\left(s_{j}\right) \neq 0$ and $\xi \cdot e_{k}\left(s_{j}, x\right) \neq 0$. Indeed, if $\xi \cdot \dot{y}\left(s_{j}\right)=0$, then $\Pi(x, \xi)$ is tangent to $C_{P I}(x)$ at $y\left(s_{j}\right)$. If $\xi \cdot e_{k}\left(s_{j}, x\right)=0$, then $\Pi(x, \xi)$ is tangent to $C_{P I}(x)$ at $y\left(s_{j}\right)$ if $k=1$ or at $y\left(s_{\text {tan }}\right)$ if $k=2$. In both cases this leads to the contradiction $\xi \in C r i t(x)$. This argument implies also that $B_{k}(x, \xi)$ is locally constant in a neighborhood of any $\xi \notin C r i t(x)$.

We now study the functions $B_{k}(x, \xi)$. Recall that $x \in U$ is fixed. By construction, $G_{\epsilon}(x, \xi) \in C^{\infty}\left(\mathbb{R}^{3}\right)$. Since $e^{i \xi \cdot x} G_{\epsilon}(x, \xi) \rightarrow B_{k}(x, \xi), \epsilon \rightarrow 0$, on $\mathbb{R}^{3} \backslash \operatorname{Crit}(x)$ and the set $\operatorname{Crit}(x)$ has Lebesgue measure zero, $B_{k}(x, \xi)$ is measurable (cf. [Lan93, p. 125]). Moreover, $B_{k}(x, \xi) \in L^{\infty}\left(\mathbb{R}^{3}\right)$ because the functions $G_{\epsilon}(x, \xi)$ are uniformly bounded on $\mathbb{R}^{3}$ as $\epsilon \rightarrow 0$.

To compute $B_{k}(x, \xi)$ suppose first that the x-ray source is fixed at $y\left(s_{0}\right)$ for some $s_{0} \in I_{P I}(x)$. Project stereographically the upper and lower turns of the spiral onto the detector plane as shown in Figure 1, left panel. It is assumed that the detector plane is parallel to the axis of the spiral and is tangential to the cylinder $y_{1}^{2}+y_{2}^{2}=R^{2}$ (cf. (2.1)) at the point opposite to the source. Thus, the distance between $y\left(s_{0}\right)$ and the detector plane is $2 R$. Let the $d_{2}$-axis be parallel to the axis of the spiral and the $d_{1}$-axis be perpendicular to it. This gives the following parametric curves:

$$
d_{1}(s)=2 R \frac{\sin \left(s-s_{0}\right)}{1-\cos \left(s-s_{0}\right)}, \quad d_{2}(s)=\frac{h}{\pi} \frac{s-s_{0}}{1-\cos \left(s-s_{0}\right)}, \quad 0<\left|s-s_{0}\right|<2 \pi .
$$

The top and bottom curves are denoted $\Gamma_{t o p}$ and $\Gamma_{b o t}$, respectively (see Figure 2). The portions of $\Gamma_{t o p}$ and $\Gamma_{b o t}$ outside the range

$$
\Delta \leq s-s_{0} \leq 2 \pi-\Delta \text { or } \Delta-2 \pi \leq s-s_{0} \leq-\Delta
$$

correspond to zero $\mathrm{CB}$ data. Here $\Delta$ is determined by the radius of support of the patient: $\Delta=2 \cos ^{-1}(r / R)$ (cf. (2.2)). By assumption, $s_{0} \in I_{P I}(x)$, so $x$ is projected into the area between $\Gamma_{t o p}$ and $\Gamma_{b o t}$. Let $\hat{x}$ denote this projection. Equations (2.23) imply that the curves $\Gamma_{b o t}$ and $\Gamma_{\text {top }}$ are strictly convex. Also, $\Gamma_{\text {top }}$ approaches $L_{0}$ from above as $s \rightarrow s_{0}^{+}$(in this case $d_{1}(s) \rightarrow+\infty$ ); $\Gamma_{b o t}$ approaches $L_{0}$ from below as $s \rightarrow s_{0}^{-}\left(d_{1}(s) \rightarrow-\infty\right)$. Here $L_{0}$ denotes the intersection of the plane containing $y\left(s_{0}\right)$ and vectors $\dot{y}\left(s_{0}\right), \ddot{y}\left(s_{0}\right)$ with the detector plane. 


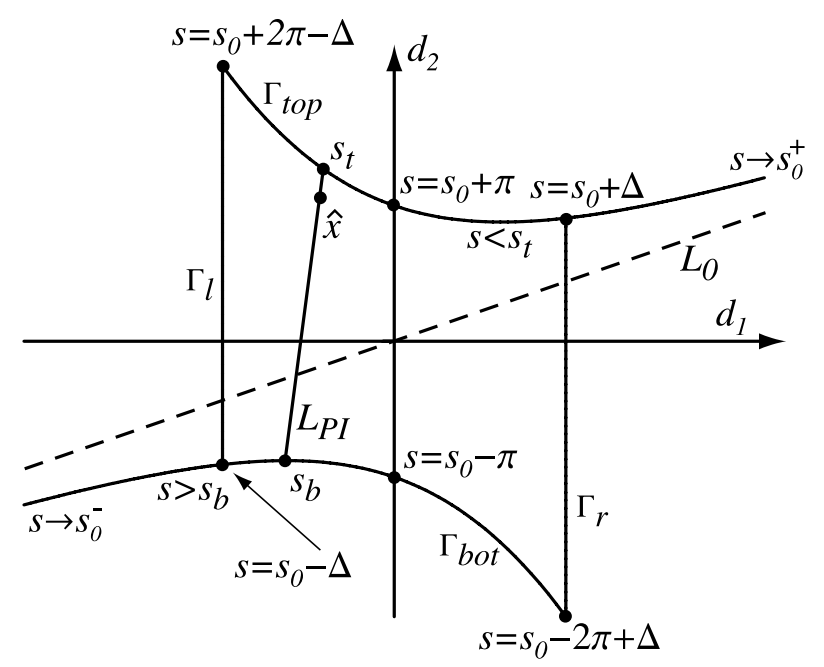

FIG. 2. Detector plane.

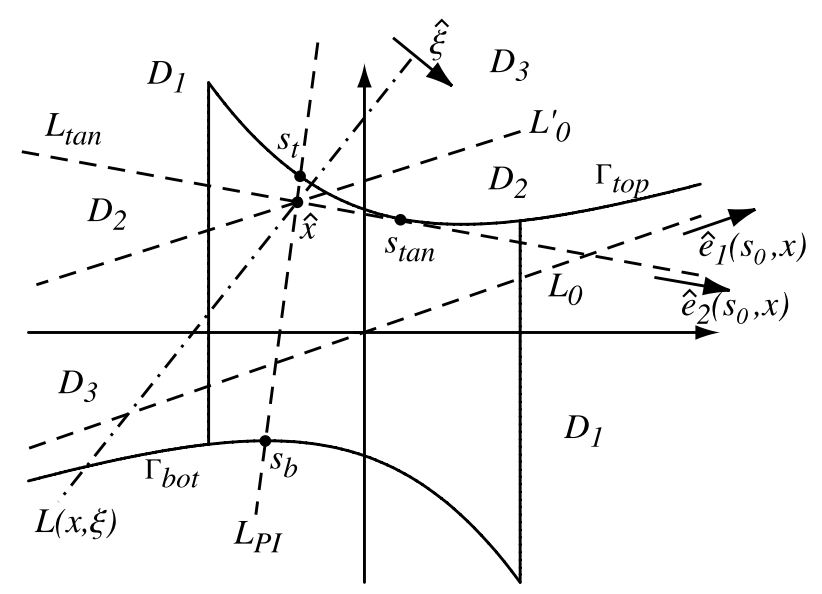

FIG. 3. Detector plane with various lines through $\hat{x}$ shown.

Consider various lines through $\hat{x}$ (see Figure 3 ). $L_{P I}$ denotes the intersection of the plane containing $x, y\left(s_{0}\right), y\left(s_{b}(x)\right), y\left(s_{t}(x)\right)$ with the detector plane. Note that if $\Gamma_{b o t}$ and $\Gamma_{t o p}$ are intersected by a vertical line (i.e., parallel to the $d_{2}$-axis), then the difference between values of the parameter $s$ at the two points of intersection is exactly $s_{2}-s_{1}=2 \pi$. By construction, $s_{t}(x)-s_{b}(x)<2 \pi$. Therefore, $L_{P I}$ has positive slope in Figure 2. $L_{\text {tan }}$ denotes the intersection of the plane containing $x, y\left(s_{0}\right)$, and tangent to $C_{P I}(x)$, with the detector plane. Obviously, the property of tangency is preserved under the stereographic projection described here. Thus, $s_{\text {tan }}$ shown in Figure 3 is exactly the same as that provided by (2.7). This observation and the properties of $\Gamma_{b o t}$ and $\Gamma_{t o p}$ imply that the solution $s_{t a n} \in I_{P I}(x)$ to $(2.7)$ exists and is unique. Another conclusion we can draw from Figure 3 is that $\check{x} \notin L_{0}$ (that is, $s_{0} \neq \check{s}(x)$ ) implies $s_{\text {tan }} \neq s_{0}$. $L_{0}^{\prime}$ is the line through $\hat{x}$ and parallel to $L_{0}$. Finally, $L(x, \xi)$ is the intersection of $\Pi(x, \xi) \ni y\left(s_{0}\right)$ with the detector plane. Clearly, there is one-toone correspondence between the planes $\Pi(x, \xi)$, where $\xi$ satisfies $\xi \cdot\left(x-y\left(s_{0}\right)\right)=0$ 
and the lines $L(x, \xi)$. The lines $L_{P I}, L_{t a n}$, and $L_{0}^{\prime}$ split the detector plane into three conical regions: $D_{j}, j=1,2,3$. If $L(x, \xi) \subset D_{1}, \Pi(x, \xi) \cap C_{P I}(x)$ contains only one point- $y\left(s_{0}\right)$. If $L(x, \xi) \subset D_{2}$, there are three points of intersection of $\Pi(x, \xi)$ with $C_{P I}(x)$. They correspond to values of the parameter $s=s_{1,2,3}$ that satisfy $s_{1}=s_{0}<s_{2}<s_{3}<s_{t}(x)$. Recall that in this region the slope of $L(x, \xi)$ is smaller than that of $L_{0}$. If $L(x, \xi) \subset D_{3}$ (an example of such a line is shown in Figure 3), then again there are three points of intersection of $\Pi(x, \xi)$ with $C_{P I}(x)$, and $s_{b}(x)<$ $s_{1}<s_{2}=s_{0}<s_{3}<s_{t}(x)$.

Summarizing, we can make the following conclusions. First, the condition $\xi \in \Xi_{1}$ is equivalent to $L(x, \xi) \subset D_{1}$, and in this case $C_{P I}(x) \cap \Pi(x, \xi)$ consists of one point. Second, the condition $\xi \in \operatorname{Crit}(x)$ is equivalent to $L(x, \xi) \in\left\{L_{0}^{\prime}, L_{t a n}, L_{P I}\right\}$. And, finally, by considering the cases still unaccounted for, we see that the condition $\xi \in \Xi_{3}$ is equivalent to $L(x, \xi) \subset D_{2}$ or $D_{3}$. When this happens, $C_{P I}(x) \cap \Pi(x, \xi)$ consists of precisely three points.

In order to compute the value of the sum in (2.22) we need a simplifying argument. First of all, since $\xi \cdot \beta\left(s_{j}, x\right)=0,(2.6)$ immediately implies

$$
\operatorname{sgn}\left(\xi \cdot \dot{y}\left(s_{j}\right)\right)=\operatorname{sgn}\left(\xi \cdot e_{1}\left(s_{j}, x\right)\right) .
$$

Let $\hat{\xi}$ be a nonzero vector in the detector plane perpendicular to $L(x, \xi)$ and pointing into the same half-space as $\xi$; that is, $\xi \cdot \hat{\xi}>0$. Fix any nonzero vector $e \in \mathbb{R}^{3}$ perpendicular to $\beta\left(s_{0}, x\right)$, and let $L$ be the line in the intersection of $\Pi\left(x, \beta\left(s_{0}, x\right) \times e\right)$ with the detector plane. Analogously, $\hat{e}$ denotes a vector in the detector plane parallel to $L$ and with the property $e \cdot \hat{e}>0$. We claim that

$$
\operatorname{sgn}(\xi \cdot e)=\operatorname{sgn}(\hat{\xi} \cdot \hat{e})
$$

Indeed, let $d_{0}$ be the unit vector perpendicular to the detector plane and pointing from the source position $y\left(s_{0}\right)$ towards the detector. This implies $\beta\left(s_{0}, x\right) \cdot d_{0}>0$. It is easy to check that

$$
\hat{\xi}=d_{0} \times\left(\xi \times d_{0}\right), \hat{e}=d_{0} \times\left(e \times \beta\left(s_{0}, x\right)\right) .
$$

Therefore,

$$
\hat{e} \cdot \hat{\xi}=\left(\xi \times d_{0}\right) \cdot\left(e \times \beta\left(s_{0}, x\right)\right)=\left(\beta\left(s_{0}, x\right) \cdot d_{0}\right)(e \cdot \xi),
$$

and (2.26) follows. In (2.28) we have used that $\beta\left(s_{0}, x\right) \cdot \xi=0$. Combining (2.25) and (2.26) gives

$$
\begin{aligned}
\operatorname{sgn}\left(\xi \cdot \dot{y}\left(s_{j}\right)\right) \operatorname{sgn}\left(\xi \cdot e_{2}\left(s_{j}, x\right)\right) & =\operatorname{sgn}\left(\xi \cdot e_{1}\left(s_{j}, x\right)\right) \operatorname{sgn}\left(\xi \cdot e_{2}\left(s_{j}, x\right)\right) \\
& =\operatorname{sgn}\left(\hat{\xi} \cdot \hat{e}_{1}\left(s_{j}, x\right)\right) \operatorname{sgn}\left(\hat{\xi} \cdot \hat{e}_{2}\left(s_{j}, x\right)\right) .
\end{aligned}
$$

For convenience, vectors $\hat{e}_{1,2}\left(s_{0}, x\right)$ are shown in Figure 3 . We use the notation $s_{0}$ instead of $s_{j}$ to denote a generic location of the source from which the stereographic projection is performed.

Let us discuss how vectors $\hat{e}_{1}\left(s_{0}, x\right)$ and $\hat{e}_{2}\left(s_{0}, x\right)$ should be drawn in Figure 3. By construction, $\dot{y}\left(s_{0}\right)$ is parallel to the detector plane; that is, $d_{0} \cdot \dot{y}\left(s_{0}\right)=0$. From (2.6) and (2.27),

$$
\begin{aligned}
\hat{e}_{1}\left(s_{0}, x\right) & =\frac{1}{c} d_{0} \times\left\{\left[\left(\beta\left(s_{0}, x\right) \times \dot{y}\left(s_{0}\right)\right) \times \beta\left(s_{0}, x\right)\right] \times \beta\left(s_{0}, x\right)\right\} \\
& =\frac{1}{c} d_{0} \times\left[\dot{y}\left(s_{0}\right) \times \beta\left(s_{0}, x\right)\right]=\frac{1}{c}\left(d_{0} \cdot \beta\left(s_{0}, x\right)\right) \dot{y}\left(s_{0}\right),
\end{aligned}
$$


where $c>0$ is the denominator in $(2.6)$ and $d_{0} \cdot \beta\left(s_{0}, x\right)>0$. Hence, $\hat{e}_{1}\left(s_{0}, x\right)$ is parallel to $\dot{y}\left(s_{0}\right)$. Therefore, we should draw $\hat{e}_{1}\left(s_{0}, x\right)$ parallel to $L_{0}$ and pointing upward (i.e., in the direction of increasing $s$ ). From (2.6), (2.8), and (2.9) it follows that $\hat{e}_{1}\left(s_{0}, x\right)=\hat{e}_{2}\left(s_{0}, x\right)$ if $s_{0}=\check{s}(x)$. By construction, $e_{2}(s, x)$ and, consequently, $\hat{e}_{2}(s, x)$ are continuous. Clearly, $\hat{e}_{2}(s, x) \neq 0$, because otherwise $\beta\left(s_{0}, x\right) \perp d_{0}$, which contradicts $x \in U$. Hence $\hat{e}_{2}\left(s_{0}, x\right)$ should be drawn as parallel to $L_{\text {tan }}$ and pointing in the direction that would coincide with $\hat{e}_{1}\left(s_{0}, x\right)$ when $s_{0} \rightarrow \check{s}(x)$ (in this case $s_{\text {tan }} \rightarrow \check{s}(x)$ and $\left.L_{\text {tan }} \rightarrow L_{0}\right)$.

From (2.22), (2.25), and the discussion preceding it regarding the number of points in $\Pi(x, \xi) \cap C_{P I}(x)$ we immediately get the formula for $B_{1}(x, \xi)$ in (2.12). Consider now $B_{2}(x, \xi)$. Suppose $\xi \in \Xi_{1}$. Since in this case $\Pi(x, \xi) \cap C_{P I}(x)$ consists of only one point, $L(x, \xi) \subset D_{1}$ and $\operatorname{sgn}\left(\hat{\xi} \cdot \hat{e}_{1}\left(s_{1}, x\right)\right)=\operatorname{sgn}\left(\hat{\xi} \cdot \hat{e}_{2}\left(s_{1}, x\right)\right)$. Hence, from $(2.22)$ and $(2.29)$,

$$
B_{2}(x, \xi)=\operatorname{sgn}\left(\xi \cdot \dot{y}\left(s_{1}\right)\right) \operatorname{sgn}\left(\xi \cdot e_{2}\left(s_{1}, x\right)\right)=1, \quad \xi \in \Xi_{1} .
$$

If $\xi \in \Xi_{3}$, there are three points in $\Pi(x, \xi) \cap C_{P I}(x)$ corresponding to $s_{1}<s_{2}<s_{3}$. Take $s_{0}=s_{1}$. In this event $L(x, \xi) \subset D_{2}$ and $\operatorname{sgn}\left(\hat{\xi} \cdot \hat{e}_{1}\left(s_{1}, x\right)\right)=-\operatorname{sgn}\left(\hat{\xi} \cdot \hat{e}_{2}\left(s_{1}, x\right)\right)$. Using (2.29) we conclude

$$
\operatorname{sgn}\left(\xi \cdot \dot{y}\left(s_{1}\right)\right) \operatorname{sgn}\left(\xi \cdot e_{2}\left(s_{1}, x\right)\right)=-1, \quad \xi \in \Xi_{3} .
$$

If $s_{0}=s_{2}, L(x, \xi)$ belongs to the region $D_{3}$ (example of such a line is shown in Figure 3$)$ and $\operatorname{sgn}\left(\hat{\xi} \cdot \hat{e}_{1}\left(s_{1}, x\right)\right)=\operatorname{sgn}\left(\hat{\xi} \cdot \hat{e}_{2}\left(s_{1}, x\right)\right)$. Therefore, from (2.29),

$$
\operatorname{sgn}\left(\xi \cdot \dot{y}\left(s_{2}\right)\right) \operatorname{sgn}\left(\xi \cdot e_{2}\left(s_{2}, x\right)\right)=1, \quad \xi \in \Xi_{3} .
$$

If $s_{0}=s_{3}$, the situation is similar to the case $s_{0}=s_{1}$ (only now $\hat{x}$ will appear under the line $\left.L_{0}\right)$ and $\operatorname{sgn}\left(\hat{\xi} \cdot \hat{e}_{1}\left(s_{3}, x\right)\right)=-\operatorname{sgn}\left(\hat{\xi} \cdot \hat{e}_{2}\left(s_{3}, x\right)\right)$. Therefore, from (2.29),

$$
\operatorname{sgn}\left(\xi \cdot \dot{y}\left(s_{3}\right)\right) \operatorname{sgn}\left(\xi \cdot e_{2}\left(s_{3}, x\right)\right)=-1, \quad \xi \in \Xi_{3} .
$$

Using (2.32)-(2.34) in (2.22) and (2.31), we prove the formula for $B_{2}(x, \xi)$ in (2.12).

We establish now the properties of $e_{2}(s, x)$ and $s_{\text {tan }}$ which were used above (see the paragraph below (2.9)). Note first that if $s \in\left(s_{b}(x), s_{t}(x)\right) \backslash\{\check{s}(x)\}$, then the denominator in $(2.9)$ is never zero because $\left(x-y\left(s_{\text {tan }}\right)\right) \times(x-y(s)) \neq 0$. Indeed, otherwise $x-y\left(s_{\text {tan }}\right)$ and $x-y(s)$ would be parallel, the line through $y\left(s_{\text {tan }}\right)$, and $y(s)$ would contain $x$, leading to $\left\{s, s_{\tan }\right\}=\left\{s_{b}(x), s_{t}(x)\right\}$ and a contradiction. Recall that $s \neq s_{\text {tan }}$ unless $s=\breve{s}(x)$. Differentiating (2.7) with respect to $s$ we conclude that $s_{\text {tan }}=s_{\text {tan }}(s, x)$ is smooth unless

$$
\left[\left(x-y\left(s_{\text {tan }}\right)\right) \times(x-y(s))\right] \cdot \ddot{y}\left(s_{\text {tan }}\right)=0, \quad s_{\text {tan }} \neq s .
$$

Combining (2.7) with (2.35) we see that this is possible only if $x$ and $y(s)$ belong to the plane through $y\left(s_{\tan }\right)$ and are parallel to either $\dot{y}\left(s_{\tan }\right), \ddot{y}\left(s_{\tan }\right)$ or $\left(x-y\left(s_{\tan }\right)\right) \|$ $(x-y(s))$. The first assumption leads to $s=s_{\text {tan }}=\check{s}(x)$, and the second leads to $\left\{s, s_{\text {tan }}\right\}=\left\{s_{b}(x), s_{t}(x)\right\}$. Hence, $s_{\text {tan }}(s, x) \in C^{\infty}\left(\left(s_{b}(x), s_{t}(x)\right) \backslash\{\check{s}(x)\}\right)$ for every fixed $x \in U$. The continuity of $s_{\tan }(s, x)$ at $s_{b}(x), \check{s}(x), s_{t}(x)$ can be easily inferred from Figure 3. For example, if $s \rightarrow \check{s}(x)$, the point $\hat{x}$ slides along $L_{P I}$ towards $L_{0}$ and $s_{\text {tan }} \rightarrow \check{s}(x)$. If $s \rightarrow s_{b}(x)$, then $\hat{x}$ approaches $\Gamma_{\text {top }}$ (at the same time the image of $y\left(s_{b}(x)\right)$ on the detector plane moves to the left and down as it approaches $L_{0}$ ) and 
$s_{\text {tan }} \rightarrow s_{t}(x)$. Similarly, if $s \rightarrow s_{t}(x)$, then $\hat{x}$ approaches $\Gamma_{b o t}$ (at the same time the image of $y\left(s_{t}(x)\right)$ moves to the right and up as it approaches $\left.L_{0}\right)$ and $s_{t a n} \rightarrow s_{b}(x)$. Of course, these limits can be justified rigorously. Assuming without loss of generality that $\check{s}(x)=0$, considering, for example, $s \rightarrow \check{s}(x)$, expanding (2.7) in the Taylor series, and considering the first nonzero term, we get

$$
\frac{[(x-y(0)) \times \dot{y}(0)] \cdot \dddot{y}(0)}{6}\left(s_{\text {tan }}-s\right)^{2}\left(2 s_{\text {tan }}+s\right)+O\left(s^{4}\right)=0 .
$$

The leading coefficient in (2.36) is not zero. Indeed, by construction $x-y(0)=$ $a \dot{y}(0)+b \ddot{y}(0)$ for some scalars $a$ and $b$. Moreover, $x \in U$ implies $b \neq 0$ and, using the properties of the spiral,

$$
[(x-y(0)) \times \dot{y}(0)] \cdot \dddot{y}(0)=b[\ddot{y}(0) \times \dot{y}(0)] \cdot \dddot{y}(0) \neq 0 .
$$

In view of (2.39) below, the solution $s_{\text {tan }}=s(1+o(1))$ is impossible, and we get from $(2.36)$ that $s_{\text {tan }}=-0.5 s(1+o(1))$, thereby confirming the conclusion that $s_{\text {tan }} \rightarrow \check{s}(x)$ if $s \rightarrow \check{s}(x)$. The other two limits can be treated similarly and they give

$$
\begin{aligned}
& s_{\text {tan }}-s_{t}(x)=O\left(\sqrt{s-s_{b}(x)}\right), s \rightarrow s_{b}(x), s>s_{b}(x), \\
& s_{\text {tan }}-s_{b}(x)=O\left(\sqrt{s_{t}(x)-s}\right), s \rightarrow s_{t}(x), s<s_{t}(x) .
\end{aligned}
$$

Our argument also implies that if $s$ stays on one side of $\check{s}(x), \hat{x}$ will never cross $L_{0}$ and, consequently, $s_{\text {tan }}$ will never become equal to $\breve{s}(x)$. Considering the limiting cases $s \rightarrow s_{b}(x)$ and $s \rightarrow s_{t}(x)$, we conclude

$$
\begin{aligned}
& \check{s}(x)<s_{\text {tan }}(s, x)<s_{t}(x) \text { if } s_{b}(x)<s<\check{s}(x), \\
& s_{b}(x)<s_{\text {tan }}(s, x)<\check{s}(x) \text { if } \check{s}(x)<s<s_{t}(x) .
\end{aligned}
$$

3. Practical implementation and numerical experiments. In this section we discuss efficient algorithms for computing $\mathcal{B}_{1,2} f$. Denoting

$$
e_{1}(s, \beta):=\frac{[\beta \times \dot{y}(s)] \times \beta}{|[\beta \times \dot{y}(s)] \times \beta|}, \quad \beta \in S^{2},
$$

rewrite $\mathcal{B}_{1} f$ as follows:

$$
\begin{aligned}
\left(\mathcal{B}_{1} f\right)(x) & :=-\frac{1}{2 \pi^{2}} \int_{I_{P I}(x)} \frac{1}{|x-y(s)|} \Psi_{1}(s, \beta(s, x)) d s, \\
\Psi_{1}(s, \beta) & :=\left.\int_{0}^{2 \pi} \frac{\partial}{\partial q} D_{f}\left(y(q), \cos \gamma \beta+\sin \gamma e_{1}(s, \beta)\right)\right|_{q=s} \frac{1}{\sin \gamma} d \gamma .
\end{aligned}
$$

Let $\Pi(\omega), \omega \in \mathbb{R}$, denote the family of planes containing $y(s)$ and let $\Pi(\omega)$ be parallel to $\dot{y}(s)$. Intersections of $\Pi(\omega)$ with the detector plane generate a family of lines $L(\omega)$ parallel to $L_{0}$ (see Figure 4, left panel). Fix any $\beta \in \Pi(\omega)$. By construction, vectors $\cos \gamma \beta+\sin \gamma e_{1}(s, \beta), 0 \leq \gamma<2 \pi$, belong to the same plane $\Pi(\omega)$. Recall that for convenience we think of vectors $\beta, e_{1}(s, \beta)$ and their linear combinations as if they are attached to $y(s)$. Let $\theta$ be a polar angle in $\Pi(\omega)$. Since $e_{1}(s, \beta) \cdot \beta=0,\left|e_{1}(s, \beta)\right|=1$, we can write (with abuse of notation)

$$
\beta=(\cos \theta, \sin \theta), \quad e_{1}(s, \beta)=(-\sin \theta, \cos \theta), \beta, e_{1}(s, \beta) \in \Pi(\omega) .
$$




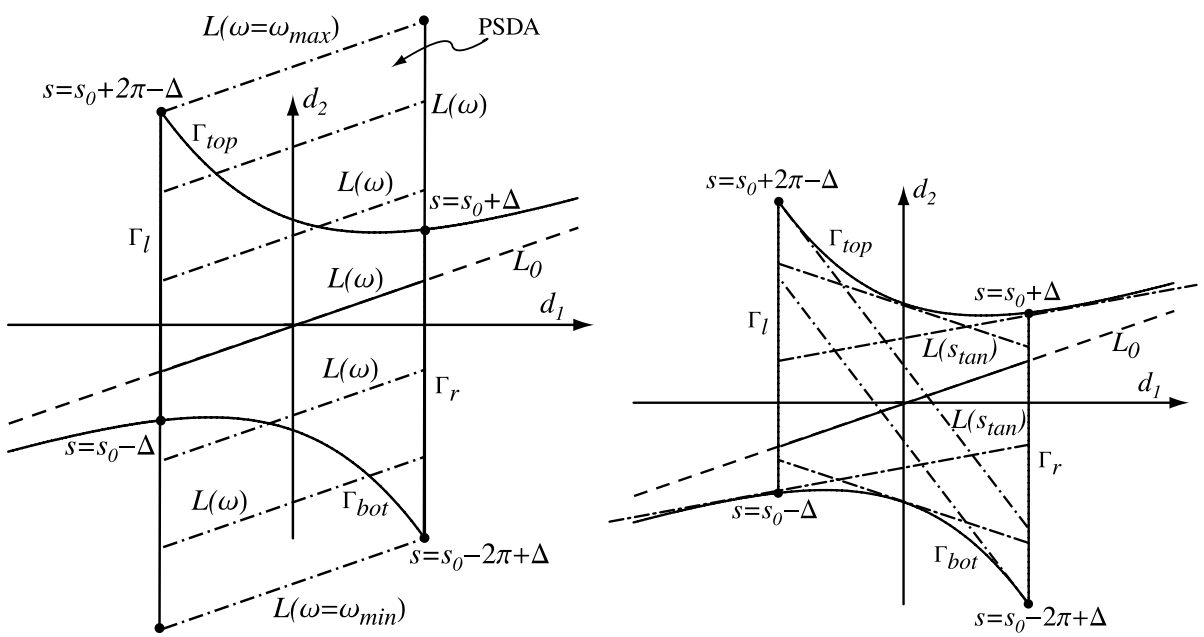

FIG. 4. Illustration of two one-parametric families of lines $L(\omega)$ and $L\left(s_{\text {tan }}\right)$ used in the numerical algorithm.

Therefore,

$$
\Psi_{1}(s, \beta)=\left.\int_{0}^{2 \pi} \frac{\partial}{\partial q} D_{f}(y(q),(\cos (\theta+\gamma), \sin (\theta+\gamma)))\right|_{q=s} \frac{1}{\sin \gamma} d \gamma, \quad \beta \in \Pi(\omega) .
$$

Equation (3.4) is of convolution type. Hence, one application of FFT to the integral in (3.4) gives values of $\Psi_{1}(s, \beta)$ for all $\beta \in \Pi(\omega)$ at once.

Calculation of $\mathcal{B}_{2} f$ can be arranged in a similar way. It follows from (2.7) that, apart from the condition $s_{\text {tan }} \in I_{P I}(x), s_{\text {tan }}$ actually depends only on $s$ and $\beta(s, x)$. Therefore, we can write

$$
\begin{aligned}
e_{2}(s, \beta) & :=\frac{[\beta \times u] \times \beta}{|[\beta \times u] \times \beta|}, \quad u=u(s, \beta), \beta \in S^{2}, \\
\Psi_{2}(s, \beta) & :=\left.\int_{0}^{2 \pi} \frac{\partial}{\partial q} D_{f}\left(y(q), \cos \gamma \beta+\sin \gamma e_{2}(s, \beta)\right)\right|_{q=s} \frac{1}{\sin \gamma} d \gamma \\
\left(\mathcal{B}_{2} f\right)(x) & :=-\frac{1}{2 \pi^{2}} \int_{I_{P I}(x)} \frac{1}{|x-y(s)|} \Psi_{2}(s, \beta(s, x)) d s .
\end{aligned}
$$

Fix $s_{\text {tan }} \in[s-2 \pi+\Delta, s+2 \pi-\Delta], s_{\text {tan }} \neq s$, and let $\Pi\left(s_{\text {tan }}\right)$ denote the plane through $y(s), y\left(s_{t a n}\right)$ containing $\dot{y}\left(s_{t a n}\right)$. If $s_{t a n}=s, \Pi\left(s_{t a n}\right)$ is determined by continuity and coincides with the plane through $y(s)$ and parallel to $\dot{y}(s), \ddot{y}(s)$. The family of lines $L\left(s_{t a n}\right)$ obtained by intersecting $\Pi\left(s_{t a n}\right)$ with the detector plane is shown in Figure 4, right panel. By construction, given any $x \in U$ with $\beta(s, x) \in \Pi\left(s_{\text {tan }}\right)$ and such that $\hat{x}$ appears to the left (right) of the point of tangency $s_{\tan }$ if $\hat{x}$ is above (below) $L_{0}$, then $s_{\text {tan }}$ used here is precisely the same as $s_{t a n}$ provided by (2.7) and (2.8). The condition that we have formulated regarding the location of $\hat{x}$ relative to $s_{\text {tan }}$ and $L_{0}$ guarantees that $s_{t a n} \in I_{P I}(x)$. Since $e_{2}(s, \beta) \cdot \beta=0,\left|e_{2}(s, \beta)\right|=1$, we can write (with abuse of notation)

$$
\beta=(\cos \theta, \sin \theta), \quad e_{2}(s, \beta)=(-\sin \theta, \cos \theta), \quad \beta, e_{2}(s, \beta) \in \Pi\left(s_{\text {tan }}\right) .
$$


Therefore,

$$
\Psi_{2}(s, \beta)=\left.\int_{0}^{2 \pi} \frac{\partial}{\partial q} D_{f}(y(q),(\cos (\theta+\gamma), \sin (\theta+\gamma)))\right|_{q=s} \frac{1}{\sin \gamma} d \gamma, \quad \beta \in \Pi\left(s_{\text {tan }}\right) .
$$

Equation (3.7) is of convolution type and one application of FFT gives values of $\Psi_{2}(s, \beta)$ for all $\beta \in \Pi\left(s_{\text {tan }}\right)$ at once. After $\Psi_{2}(s, \beta)$ has been computed, we use only the portion of it that is located to the left (right) of the point of tangency $s_{\text {tan }}$ if $L\left(s_{\text {tan }}\right)$ is above (below) $L_{0}$.

In numerical implementation of (3.2), (3.4) and (3.5), (3.7) we used bilinear interpolation to pass from a rectangular grid of points on the detector to points on the lines $L(\omega)$ and $L\left(s_{\text {tan }}\right)$ and back. As suggested by (3.4) and (3.7), the points on $L(\omega)$ and $L\left(s_{t a n}\right)$ were parametrized by polar angle in the corresponding plane.

Equations (3.2), (3.4) and (3.5), (3.7) imply that the resulting algorithm is of the FBP type. First, one computes shift-invariant filtering of a derivative of $\mathrm{CB}$ projections using (3.4) for all required $\omega: \omega_{\min } \leq \omega \leq \omega_{\max }$ (cf. Figure 4, left panel) and using (3.7) - for all $s_{t a n} \in[s-2 \pi+\Delta, s+2 \pi-\Delta]$ (cf. Figure 4, right panel). The second step is backprojection according to (3.2) and (3.5). Since $\partial / \partial q$ in (3.5) and (3.7) is a local operation, each CB projection is stored in memory as soon as it has been acquired for a short period of time for computing this derivative at a few nearby points and is never used later.

This discussion shows that for the algorithm to work the following two conditions must be satisfied. First, the detector array should be large enough to contain the parallelogram formed by the lines $\Gamma_{l}, \Gamma_{r}$ and $L\left(\omega_{\min }\right), L\left(\omega_{\max }\right)$. We will call this parallelogram the parallelogram-shaped detector array (PSDA), and its area will be denoted by $A_{P S D A}$. Thus, the size of the detector array required for the algorithm is greater than the theoretically minimum one, which is bounded by $\Gamma_{l}, \Gamma_{r}$ and $\Gamma_{t o p}, \Gamma_{b o t}$. Its area will be denoted by $A_{\min }$. The ratio of the two areas is independent of the pitch $h$ but grows as $r \rightarrow R$. For example, $A_{P S D A} / A_{\min }=1.53$ if $r / R=1 / 3$ and $A_{P S D A} / A_{\min }=1.93$ if $r / R=0.5$. Second, the segments of lines tangent to $\Gamma_{t o p}$ and $\Gamma_{b o t}$ at $s=s+2 \pi-\Delta$ and $s-2 \pi+\Delta$, respectively, and located between $\Gamma_{l}$ and $\Gamma_{r}$ should be inside the detector array. This requirement leads to the restriction $r / R \leq \cos \left(\Delta_{0} / 2\right) \approx 0.62$, where $\Delta_{0}$ is the unique solution to the equation $\tan (2 \pi-$ $\Delta)=2 \pi-\Delta$ on the interval $\pi / 2<\Delta<\pi$.

TABLE 1

Parameters of the data collection protocol.

\begin{tabular}{|c|c|c|}
\hline & $\begin{array}{c}\text { Shepp } \\
\text { phantom }\end{array}$ & $\begin{array}{c}\text { Disk } \\
\text { phantom }\end{array}$ \\
\hline$R$ (radius of the spiral) & \multicolumn{2}{|c|}{3} \\
\hline$h$ (pitch of the spiral) & \multicolumn{2}{|c|}{0.5} \\
\hline Axial span of the detector array & 1.02 & 0.96 \\
\hline Transverse span of the detector array & \multicolumn{2}{|c|}{4.74} \\
\hline Number of detector rows & \multicolumn{2}{|c|}{50} \\
\hline Number of detectors per row & \multicolumn{2}{|c|}{500} \\
\hline Number of source positions per one turn of the spiral & \multicolumn{2}{|c|}{1500} \\
\hline
\end{tabular}

Consider now two numerical experiments. Parameters of the data collection protocol are given in Table 1. Since (3.4) and (3.7) require differencing of neighboring $\mathrm{CB}$ projections, we used a somewhat higher number of sources per turn of the spiral than what is common in spiral CT (about 900-1000). 

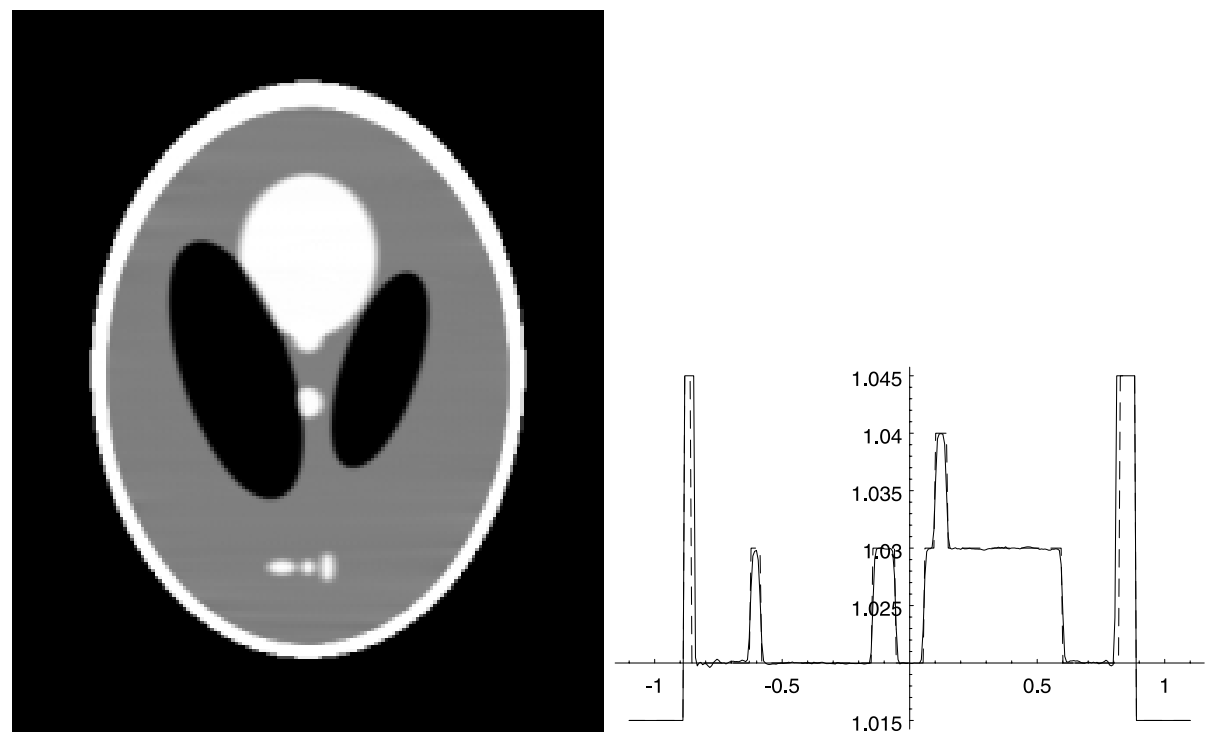

Fig. 5. Reconstruction of the 3-D Shepp phantom.

TABLE 2

Parameters of the low contrast Shepp phantom.

\begin{tabular}{|c|c|c|c|}
\hline Center & Length of axes & Rotation angle & Incremental density \\
\hline$(0,0,0)$ & $(0.9,0.69,0.92)$ & 0 & 2.0 \\
\hline$(0,0,-0.0184)$ & $(0.88,0.6624,0.874)$ & 0 & -0.98 \\
\hline$(-0.25,-0.22,0)$ & $(0.21,0.41,0.16)$ & 108 & -0.02 \\
\hline$(-0.25,0.22,0)$ & $(0.22,0.31,0.11)$ & 72 & -0.02 \\
\hline$(-0.25,0,0.35)$ & $(0.35,0.21,0.25)$ & 0 & 0.01 \\
\hline$(-0.25,0,0.1)$ & $(0.046,0.046,0.046)$ & 0 & 0.01 \\
\hline$(-0.25,-0.08,-0.605)$ & $(0.02,0.046,0.023)$ & 0 & 0.01 \\
\hline$(-0.25,0.06,-0.605)$ & $(0.02,0.046,0.023)$ & 90 & 0.01 \\
\hline$(0.625,0.06,-0.105)$ & $(0.1,0.056,0.04)$ & 90 & 0.02 \\
\hline$(0.625,0,0.1)$ & $(0.1,0.056,0.056)$ & 0 & -0.02 \\
\hline$(-0.25,0,-0.1)$ & $(0.046,0.046,0.046)$ & 0 & 0.01 \\
\hline$(-0.25,0,-0.605)$ & $(0.023,0.023,0.023)$ & 0 & 0.01 \\
\hline
\end{tabular}

In Figure 5 we show the results of reconstructing the three-dimensional (3-D) low contrast Shepp phantom of [KMS98]. For the convenience of the reader, parameters of the phantom are presented in Table 2 . In the left panel we see a vertical slice through the reconstructed image at $x_{1}=-0.25$, and in the right panel we see the graphs of exact (dashed line) and computed (solid line) values of $f$ along the vertical line $x_{1}=-0.25, x_{2}=0$. We used the grey scale window $[1.01,1.03]$ to make low contrast features visible.

In Figure 6 we see the results of reconstructing the disk phantom, which consists of six identical flattened ellipsoids (lengths of half-axes $0.75,0.75$, and 0.04 , distance between centers of neighboring ellipsoids 0.16 ). In the left panel we see the vertical slice through the reconstructed image at $x_{1}=0$, and in the right panel we see the graphs of exact (dashed line) and computed (solid line) values of $f$ along the vertical line $x_{1}=0, x_{2}=0$. To better see artifacts, the graphs of exact and computed values of $f$ along the line $x_{1}=0, x_{2}=0.7$ are presented in Figure 7 . This line is close to the outer edges of the disks, where the artifacts are more noticeable. 

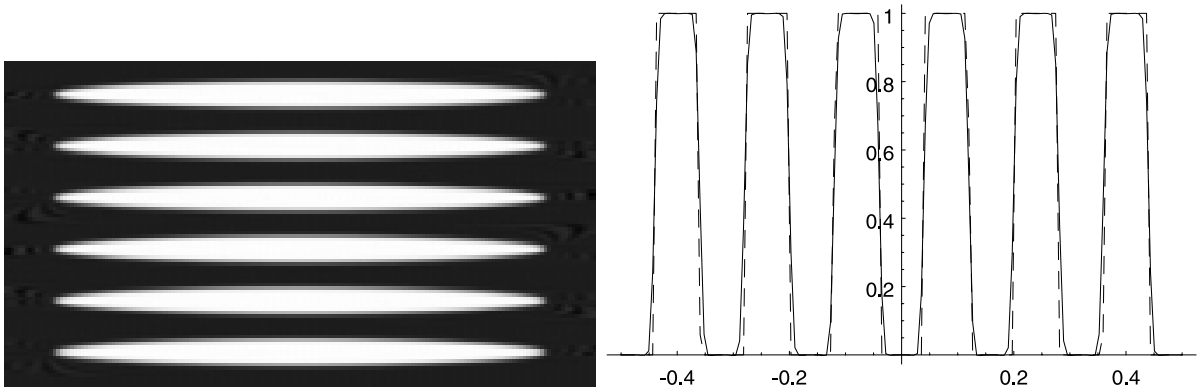

FIG. 6. Reconstruction of the disk phantom.

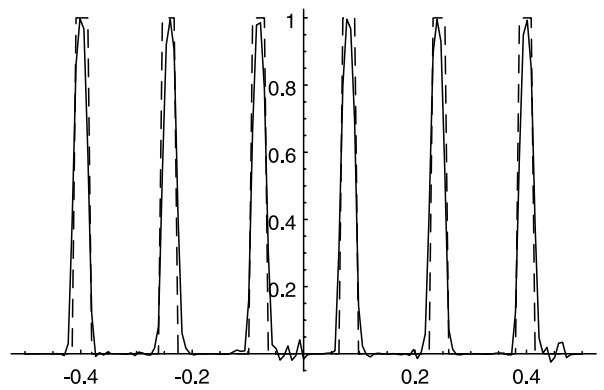

FIG. 7. Disk phantom. The graphs of exact and computed values of $f$ along the off-center line $x_{1}=0, x_{2}=0.7$.

As one can see, the algorithm produces images of good quality with a low level of artifacts. Therefore, we can conclude that our results are compatible with the hypothesis that the artifacts are only due to discretization and/or sampling errors. It is expected that further improvements in the code can reduce the artifacts as well. For example, currently the derivatives $\partial / \partial q$ in (3.4) and (3.7) are implemented using first order finite differences. The author hopes that the use of more sophisticated approximate derivatives will improve the overall image quality. An alternative approach could be to integrate by parts with respect to $s$ in (2.10) and eliminate $\partial / \partial q$ altogether by transferring it to the angular variables.

\section{REFERENCES}

$\left[\mathrm{D}^{+} 97\right]$ P. E. Danielsson, P Edmolm, J. Eriksson, And M. Seger, Towards exact 3Dreconstruction for helical cone-beam scanning of long objects. A new detector arrangement and a new completeness condition, in Proceedings of the 1997 Meeting on Fully 3D Image Reconstruction in Radiology and Nuclear Medicine (Pittsburgh), D. W. Townsend and P. E. Kinahan, eds., 1997, pp. 141-144.

[DNK00] M. Defrise, F. Noo, AND H. Kudo, A solution to the long-object problem in helical cone-beam tomography, Phys. Med. Biol., 45 (2000), pp. 623-643.

[Kat02] A. KATSEVICH, Microlocal analysis of an FBP algorithm for truncated spiral cone beam data, J. Fourier Anal. Appl., to appear.

[KMS98] H. Kudo, N. Miyagi, and T. SAito, A new approach to exact cone-beam reconstruction without Radon transform, in 1998 IEEE Nuclear Science Symposium Conference Record, IEEE, Piscataway, NJ, 1998, pp. 1636-1643.

[KND98] H. Kudo, F. Noo, AND M. Defrise, Cone-beam filtered-backprojection algorithm for truncated helical data, Phys. Med. Biol., 43 (1998), pp. 2885-2909.

[KS97] H. Kudo AND T. SAITO, An extended cone-beam reconstruction using Radon transform, 
in 1996 IEEE Medical Imaging Conference Record, IEEE, Piscataway, NJ, 1997, pp. $1693-1697$.

[Lan93] S. Lang, Real and Functional Analysis, 3rd ed., Springer-Verlag, New York, 1993.

[NKD98] F. Noo, H. Kudo, And M. Defrise, Approximate short-scan filtered-backprojection for helical CB reconstruction, in 1998 IEEE Nuclear Science Symposium Conference Record, vol. 3, IEEE, Piscataway, NJ, 1998, pp. 2073-2077.

[SNS $\left.{ }^{+} 00\right]$ S. Schaller, F. Noo, F. Sauer, K. C. Tam, G. Lauritsch, and T. Flohr, Exact Radon rebinning algorithm for the long object problem in helical cone-beam CT, IEEE Trans. Medical Imaging, 19 (2000), pp. 361-375.

[Tam95] K. C. TAM, Method and apparatus for converting cone beam x-ray projection data to planar integral and reconstructing a three-dimensional computerized tomography (CT) image of an object, US Patent 5,257,183, 1995.

[Tam97] K. C. TAM, Cone-beam imaging of a section of a long object with a short detector, in Information Processing in Medical Imaging, Lecture Notes in Comput. Sci. 1230, J. S. Duncan and G. R. Gindi, eds., Springer-Verlag, Berlin, 1997, pp. 525-530.

[TD00] H. Turbell and P.-E. Danielsson, Helical cone beam tomography, Int. J. Imaging Syst. Technology, 11 (2000), pp. 91-100. 